\title{
Long time behavior of solutions to Nernst-Planck and Debye-Hückel drift-diffusion systems
}

\author{
Piotr BILER \\ Mathematical Institute, University of Wrocław \\ pl. Grunwaldzki 2/4, 50-384 Wrocław, Poland \\ Piotr.Biler@math.uni.wroc.pl \\ and \\ Jean DOLBEAULT \\ Ceremade, U.M.R. C.N.R.S. no. 7534, \\ Université Paris IX-Dauphine \\ Pl. du Maréchal de Lattre de Tassigny, \\ 75775 Paris Cédex 16, France \\ dolbeaul@ceremade.dauphine.fr
}

March 21, 2000

\begin{abstract}
We study the convergence rates of solutions to drift-diffusion systems (arising from plasma, semiconductors and electrolytes theories) to their self-similar or steady states. This analysis involves entropytype Lyapunov functionals and logarithmic Sobolev inequalities.
\end{abstract}

Key words and phrases: drift-diffusion systems, asymptotic behavior of solutions, logarithmic Sobolev inequalities

1991 Mathematics Subject Classification: 35Q, 35B40, 35B30 


\section{Introduction}

We consider the long time asymptotics of solutions to drift-diffusion systems

$$
\begin{gathered}
u_{t}=\nabla \cdot(\nabla u+u \nabla \phi), \\
v_{t}=\nabla \cdot(\nabla v-v \nabla \phi), \\
\Delta \phi=v-u,
\end{gathered}
$$

where $u, v$ denote densities of negatively, respectively positively, charged particles. The Poisson equation (1.3) defines the electric potential $\phi$ coupling the equations (1.1)-(1.2) for the temporal evolution of charge distributions. The system (1.1)-(1.3) was formulated by W. Nernst and M. Planck at the end of the nineteenth century as a basic model for electrodiffusion of ions in electrolytes filling the whole space $\mathbb{R}^{3}$. Note that the case of multicharged particles is also covered by (1.1)-(1.3) since $u$ and $v$ denote the charge densities.

Supplemented with the no-flux boundary conditions

$$
\begin{aligned}
& \frac{\partial u}{\partial \nu}+u \frac{\partial \phi}{\partial \nu}=0, \\
& \frac{\partial v}{\partial \nu}-v \frac{\partial \phi}{\partial \nu}=0
\end{aligned}
$$

on the boundary of a bounded domain $\Omega \subset \mathbb{R}^{d}, d \leq 3$, and either

$$
\phi=0 \quad \text { on } \partial \Omega,
$$

or

$$
\phi=E_{d} *(v-u)
$$

where $E_{d}$ is the fundamental solution of the Laplacian in $\mathbb{R}^{d}$, the system (1.1)-(1.3) was also studied by P. Debye and E. Hückel in the 1920's. (1.6) signifies a conducting boundary of the container, while in the case of a bounded domain the "free" boundary condition (1.7) corresponds to a container immersed in a medium with the same dielectric constant as the solute.

These equations, together with their generalizations including e.g. an exterior potential, known as drift-diffusion Poisson systems, also appear in plasma physics and (supplemented with some mixed linear boundary conditions instead of (1.4)-(1.5)) in semiconductor device modelling. 
To determine completely the evolution, the initial conditions

$$
u(x, 0)=u_{0}(x), \quad v(x, 0)=v_{0}(x)
$$

are added. Obviously, positivity of $u_{0} \geq 0, v_{0} \geq 0$ is conserved: $u(x, t) \geq 0$, $v(x, t) \geq 0$, as well as the total charges

$$
M_{u}=\int u_{0}(x) d x=\int u(x, t) d x, \quad M_{v}=\int v_{0}(x) d x=\int v(x, t) d x .
$$

Here $M_{u}, M_{v}$ are not necessarily the same, i.e. the electroneutrality condition

$$
M_{u}=M_{v}
$$

is not, in general, required. Condition (1.10) must be satisfied in the case of the homogeneous Neumann boundary conditions $\frac{\partial \phi}{\partial \nu}=0$ (i.e. an isolated wall of the container) leading together with (1.4)-(1.5) to

$$
\frac{\partial u}{\partial \nu}=\frac{\partial v}{\partial \nu}=\frac{\partial \phi}{\partial \nu}=0
$$

Our results (Theorem 1.2 below) are valid in that case, with even a simpler proof.

The asymptotic properties of solutions to (1.1)-(1.3), (1.7) have been studied recently in [1]. The authors proved that (for $d \geq 3, M_{u}=M_{v}=1$ and $u_{0}$ and $v_{0}$ regular enough) $u, v$ tend to their self-similar asymptotic states at an algebraic rate. We improve these results by relaxing assumptions on the initial data and showing a stronger (still algebraic) decay rate, which we expect to be optimal (see Theorem 1.1 below).

In the case of a bounded domain, the convergence (with no specific speed) in the $L^{1}$-norm of $u$ and $v$ solving (1.1)-(1.5) to their corresponding steady states has been proved in [5] (as well as the $L^{\infty}$-convergence for more regular $\left.u_{0}, v_{0}\right)$. Here we prove the exponential convergence towards the steady states with a decay rate depending on $\Omega \subset \mathbb{R}^{d}, d \geq 2$, and the initial value of the entropy functional only (see Theorem 1.2 below).

Notation. The $L^{p}$-norm in $\mathbb{R}^{d}$ or $\Omega \subset \subset \mathbb{R}^{d}$ is denoted by $|\cdot|_{p}$, and inessential constants (which may vary from line to line) are denoted generically by $C$. 
Define the asymptotic states in $\mathbb{R}^{d}$ by

$$
\begin{aligned}
& u_{a s}(x, t)=\frac{M_{u}}{(2 \pi(2 t+1))^{d / 2}} \exp \left(-\frac{|x|^{2}}{2(2 t+1)}\right), \\
& v_{a s}(x, t)=\frac{M_{v}}{(2 \pi(2 t+1))^{d / 2}} \exp \left(-\frac{|x|^{2}}{2(2 t+1)}\right),
\end{aligned}
$$

where the charges of the solution $\langle u, v\rangle$ of (1.1)-(1.2) are given by (1.9), and the entropy functional by

$$
L(t)=\int u(x, t) \log \left(\frac{u(x, t)}{u_{a s}(x, t)}\right) d x+\int v(x, t) \log \left(\frac{v(x, t)}{v_{a s}(x, t)}\right) d x+\frac{1}{2}|\nabla \phi(t)|_{2}^{2} .
$$

Theorem 1.1 There exists a constant $C=C\left(d, M_{u}, M_{v}, L_{0}\right)$ such that for each solution $\langle u, v\rangle$ of (1.1)-(1.3), (1.7)-(1.8) in $\mathbb{R}^{d}, d \geq 3$, if $L(0)=L_{0}$, then for all $t \geq 0$,

$$
L(t) \leq C H(t)
$$

and

$$
\left|u(t)-u_{a s}(t)\right|_{1}^{2}+\left|v(t)-v_{a s}(t)\right|_{1}^{2}+|\nabla \phi(t)|_{2}^{2} \leq C H(t),
$$

where

$$
H(t)=\left\{\begin{array}{cc}
(2 t+1)^{-1 / 2}, & d=3, \\
(2 t+1)^{-1}(\log (2 t+1)+1), & d=4 \\
(2 t+1)^{-1}, & d>4 .
\end{array}\right.
$$

Moreover if $M_{u}=M_{v}$, then $H(t)=(2 t+1)^{-1}$ for any $d \geq 3$.

In the case of a bounded domain, define the entropy functional

$$
\begin{aligned}
W(t) & =\int u(x, t) \log u(x, t) d x-\int U(x) \log U(x) d x \\
& +\int v(x, t) \log v(x, t) d x-\int V(x) \log V(x) d x \\
& +\frac{1}{2} \int(u-v) \phi d x-\frac{1}{2} \int(U-V) \Phi d x,
\end{aligned}
$$


for the solution $\langle u, v, \phi\rangle$ of (1.1)-(1.5), (1.6) or (1.7), (1.8) and the unique steady state $\langle U, V, \Phi\rangle$ of the Debye-Hückel system with

$$
M_{u}=\int U(x) d x, \quad M_{v}=\int V(x) d x .
$$

Note that for the condition (1.6) the fifth and the sixth terms in $W(t)$ take the form $\frac{1}{2}|\nabla \phi|_{2}^{2}-\frac{1}{2}|\nabla \Phi|_{2}^{2}$.

Theorem 1.2 If $d \geq 2$, then there exist two constants $\lambda=\lambda(\Omega)>0$ and $C=$ $C\left(M_{u}, M_{v}, W_{0}\right)$ such that for each solution $\langle u, v, \phi\rangle$ of (1.1)-(1.6), (1.8) in a bounded uniformly convex domain $\Omega$, if $W(0)=W_{0}$, then for all $t \geq 0$,

$$
W(t) \leq W(0) e^{-\lambda t}
$$

and

$$
|u(t)-U|_{1}^{2}+|v(t)-V|_{1}^{2}+|\nabla(\phi-\Phi)|_{2}^{2} \leq C e^{-\lambda t} .
$$

\section{Proof of Theorem $\mathbf{1 . 1}$}

We begin with a rescaling of the system (1.1)-(1.3) which will lead to a system with a quadratic confinement potential, and therefore (eliminating the dispersion) to the expected exponential convergence to the steady states. This idea was applied in [8] and [7], as well as in [1], to a variety of problems ranging from kinetic equations to porous media equations.

Let $\bar{x} \in \mathbb{R}^{d}, \tau>0$, be the new variables defined by

$$
\bar{x}=\frac{x}{R(t)}, \quad \tau=\log R(t), \quad R(t)=(2 t+1)^{1 / 2},
$$

and consider the rescaled functions $\bar{u}, \bar{v}, \bar{\phi}$ such that

$$
\begin{aligned}
& u(x, t)=\frac{1}{R^{d}(t)} \bar{u}(\bar{x}, \tau), \\
& v(x, t)=\frac{1}{R^{d}(t)} \bar{v}(\bar{x}, \tau), \\
& \phi(x, t)=\bar{\phi}(\bar{x}, \tau) .
\end{aligned}
$$


This whole section will deal with the rescaled system, so omitting the bars over $x, u, v, \phi$ will not lead to confusions with the original system, which now takes, after rescaling, the form

$$
\begin{gathered}
u_{\tau}=\nabla \cdot(\nabla u+u x+u \nabla \phi), \\
v_{\tau}=\nabla \cdot(\nabla v+v x-v \nabla \phi), \\
\Delta \phi=e^{-\tau(d-2)}(v-u) .
\end{gathered}
$$

The scaling (2.2) preserves the $L^{1}$-norms, so the rescaled initial data $u_{0}, v_{0}$ still satisfy

$$
M_{u}=\int u_{0}(x) d x=\int u(x, \tau) d x, \quad M_{v}=\int v_{0}(x) d x=\int v(x, \tau) d x .
$$

Denote by $\left\langle u_{\infty}, v_{\infty}\right\rangle$ the steady state of (2.3)-(2.4), that is

$$
\begin{aligned}
& u_{\infty}(x)=\frac{M_{u}}{(2 \pi)^{d / 2}} \exp \left(-\frac{|x|^{2}}{2}\right), \\
& v_{\infty}(x)=\frac{M_{v}}{(2 \pi)^{d / 2}} \exp \left(-\frac{|x|^{2}}{2}\right) .
\end{aligned}
$$

Of course, going back to the original variables $x, t,\left\langle u_{\infty}, v_{\infty}\right\rangle$ corresponds to the asymptotic state $\left\langle u_{a s}, v_{a s}\right\rangle$ defined by (1.11)-(1.12). Writing $\phi=\beta \psi$ with $\beta=\beta(\tau)=e^{-\tau(d-2)} \rightarrow 0$ as $\tau \rightarrow+\infty$, we introduce the relative entropy

$$
W(\tau)=\int u \log \left(\frac{u}{u_{\infty}}\right) d x+\int v \log \left(\frac{v}{v_{\infty}}\right) d x+\frac{\beta}{2}|\nabla \psi|_{2}^{2}
$$

corresponding to the original entropy functional $L$ in (1.13). The evolution of $W$ is given by

$$
\frac{d W}{d \tau}=-\int u\left|\nabla\left(\log \frac{u}{U}\right)\right|^{2} d x-\int v\left|\nabla\left(\log \frac{v}{V}\right)\right|^{2} d x-\left(\frac{d}{2}-1\right) \beta|\nabla \psi|_{2}^{2},
$$

with $U, V$ denoting the local Maxwellians

$$
U(x, \tau)=M_{u} \frac{\exp \left(-\frac{1}{2}|x|^{2}-\phi(x, \tau)\right)}{\int \exp \left(-\frac{1}{2}|y|^{2}-\phi(y, \tau)\right) d y},
$$




$$
V(x, \tau)=M_{v} \frac{\exp \left(-\frac{1}{2}|x|^{2}+\phi(x, \tau)\right)}{\int \exp \left(-\frac{1}{2}|y|^{2}+\phi(y, \tau)\right) d y},
$$

so that $\nabla U / U=-(x+\nabla \phi), \nabla V / V=-(x-\nabla \phi)$. Using the notation

$$
J=\frac{1}{2} \int u\left|\frac{\nabla u}{u}+x\right|^{2} d x+\frac{1}{2} \int v\left|\frac{\nabla v}{v}+x\right|^{2} d x
$$

(2.10) can be rewritten as

$$
\begin{aligned}
\frac{d W}{d \tau}=- & J-2 \int(\nabla u-\nabla v) \cdot \nabla \phi d x-2 \int(u-v) x \cdot \nabla \phi d x \\
& \quad-\int(u+v)|\nabla \phi|^{2} d x-\left(\frac{d}{2}-1\right) \beta|\nabla \psi|_{2}^{2} \\
= & -2 J-\beta^{2} \int(u+v)|\nabla \psi|^{2} d x-2 \beta|u-v|_{2}^{2}+\left(\frac{d}{2}-1\right) \beta|\nabla \psi|_{2}^{2} .
\end{aligned}
$$

The quantity $J$ in (2.13) can be estimated from below using the Gross logarithmic Sobolev inequality

$$
\int f \log \left(\frac{f}{|f|_{1}}\right) d x+d\left(1+\frac{1}{2} \log (2 \pi a)\right)|f|_{1} \leq \frac{a}{2} \int \frac{|\nabla f|^{2}}{f} d x
$$

valid for each $a>0$, see e.g. [11] or a thorough discussion of different versions of logarithmic Sobolev inequalities in [2]. (2.15) becomes an equality if and only if $f(x)=C \exp \left(-|x|^{2} /(2 a)\right)$ (up to a translation).

Taking $a=1$ in (2.15), the relation (2.14) leads to

$$
-\left(\frac{d W}{d \tau}+2 W\right) \geq 2 \beta|u-v|_{2}^{2}-\beta \frac{d}{2}|\nabla \psi|_{2}^{2} \geq-C \beta\left(M_{u}+M_{v}\right)^{2}
$$

with a constant $C=C(d)=\frac{2}{d}\left(\frac{d-2}{4}\right)^{(d-2) / 2} \Sigma^{d / 2}$, because by the Hardy-Littlewood-Sobolev inequality and an interpolation

$$
|\nabla \psi|_{2}^{2} \leq \Sigma|u-v|_{2 d /(d+2)}^{2} \leq \Sigma|u-v|_{1}^{4 / d}|u-v|_{2}^{2-4 / d} \leq \frac{4}{d}|u-v|_{2}^{2}+C|u-v|_{1}^{2} .
$$

Clearly, (2.16) implies

$$
\frac{d}{d \tau}\left(e^{2 \tau} W(\tau)\right) \leq C\left(M_{u}+M_{v}\right)^{2} e^{\tau(4-d)}
$$


and, after one integration, we obtain

$$
W(\tau) \leq\left(W(0) e^{-\tau}+C\left(M_{u}+M_{v}\right)^{2}\right) e^{-\tau}
$$

in the case $d=3$,

$$
W(\tau) \leq\left(W(0)+C\left(M_{u}+M_{v}\right)^{2} \tau\right) e^{-2 \tau}
$$

if $d=4$, and finally for all $d>4$

$$
W(\tau) \leq\left(W(0)+C\left(M_{u}+M_{v}\right)^{2}\right) e^{-2 \tau} .
$$

Since from the Csiszár-Kullback inequality (cf. (1.9) in [2], App. D in [7], [6] or [10]) $W(\tau)$ controls the $L^{1}$-norm of $u-u_{\infty}$ and $v-v_{\infty}$, we get the same decay rates as in (2.17)-(2.19) for

$$
\left|u(\tau)-u_{\infty}\right|_{1}^{2}+\left|v(\tau)-v_{\infty}\right|_{1}^{2}+\beta|\nabla \psi(\tau)|_{2}^{2} \leq 2\left(\max \left(M_{u}, M_{v}\right)+1\right) W(\tau) .
$$

Returning to the original variables $x, t$, this implies, of course, the estimates (1.14)-(1.15) of Theorem 1.1 in the general case.

In the electroneutrality case (1.10): $M_{u}=M_{v}$, since $u_{\infty}=v_{\infty}$, so for $d=3$, $|u-v|_{1}^{2}=\left|u-u_{\infty}+v_{\infty}-v\right|_{1}^{2} \leq C e^{-\tau}$. Next, a modification of (2.16) reads

$$
\frac{d}{d \tau}\left(e^{2 \tau} W(\tau)\right) \leq C e^{2 \tau} \beta|u-v|_{1}^{2} \leq C,
$$

and this leads to $W(\tau) \leq C(1+\tau) e^{-2 \tau}$. Inserting this into (2.20) and (2.16) once again implies

$$
\frac{d}{d \tau}\left(e^{2 \tau} W(\tau)\right) \leq C(1+\tau) e^{-\tau},
$$

so that $W(\tau) \leq C e^{-2 \tau}$. If $d=4$, the same reasoning once again applies providing also the same improved decay rate.

Remark 2.1 Note that the constant $C$ in (1.15) depends on $d, M_{u}, M_{v}$ and $L(0)$ only, and is independent of e.g. $\left|u_{0}\right|_{r},\left|v_{0}\right|_{r}$ with some $r>d / 2-$ as it was in fact in [1]. Conditions like $\left|u_{0}\right|_{r}+\left|v_{0}\right|_{r}<\infty$ are sufficient for (local in 
time) existence of solutions to the considered systems (cf. Theorem 2 in [5]), but they can be relaxed - as it was done for a related parabolic-elliptic system describing the gravitational interaction of particles in [4]. Thus, compared to [1], Theorem 1.1 gives not only an improvement of the exponents but also gets rid of the unnecessary dependence on quantities other than $L(0), M_{u}$, $M_{v}$. We do not know if the exponents in Theorem 1.1 are optimal, but such a conjecture is supported by the calculations in the proof of the following

Proposition 2.2 There exists a constant $\lambda>0$ depending only on $d$ with $\lambda \geq \lambda(d)=(d-2)\left(\sqrt{(d-1)^{2}+3}-(d-1)\right)$, such that

$$
W(\tau) \leq W(0) e^{-\lambda \tau}
$$

and hence

$$
L(t) \leq L(0)(2 t+1)^{-\lambda / 2}
$$

for each solution $\langle u, v\rangle$ to the Nernst-Planck system.

Remark 2.3 The interest of this proposition is that the constants controlling the convergence of $W(t), L(t)$, and hence $\left|u-u_{a s}\right|_{1},\left|v-v_{a s}\right|_{1}$ in (1.15), depend on the initial values of $W(0), L(0)$ only (and not on $|u|_{1}=M_{u},|v|_{1}=$ $M_{v}$, which are quantities not comparable with, say, $\int u \log u d x, \int v \log v d x$ in the whole $\mathbb{R}^{d}$ space case). However, the exponent $\lambda$ - which is evaluated explicitly - is not as good as the one in Theorem 1.1.

Proof of Proposition 2.2. Using (2.9), (2.13), (2.14), we may write for any positive $\lambda$

$$
\begin{aligned}
-\left(\frac{d W}{d \tau}+\lambda W\right) & =\lambda\left(J-\int u \log \left(\frac{u}{u_{\infty}}\right)-\int v \log \left(\frac{v}{v_{\infty}}\right)\right) \\
& +(2-\lambda) J+B+2 E-\mu F
\end{aligned}
$$

where

$$
\begin{aligned}
& B=\beta^{2} \int(u+v)|\nabla \psi|^{2} d x, \\
& E=\beta|u-v|_{2}^{2}, \\
& F=\left(\frac{d}{2}-1\right) \beta|\nabla \psi|_{2}^{2}, \\
& \mu=1+\frac{\lambda}{d-2} .
\end{aligned}
$$


Observe that if we define

$$
G_{1}=\int u\left(\frac{\nabla u}{u}+x\right) \cdot \nabla \phi d x, \quad G_{2}=\int v\left(\frac{\nabla v}{v}+x\right) \cdot \nabla \phi d x
$$

then

$$
G_{1}-G_{2}=\int \nabla(u-v) \cdot \nabla \phi d x+\int(u-v)(x \cdot \nabla \phi) d x=E-F .
$$

Define now

$$
\begin{gathered}
f_{1}=\sqrt{2-\lambda} \cdot \sqrt{u}\left(\frac{\nabla u}{u}+x\right), \quad g_{1}=\sqrt{u} \nabla \phi, \\
f_{2}=\sqrt{2-\lambda} \cdot \sqrt{v}\left(\frac{\nabla v}{v}+x\right), \quad g_{2}=\sqrt{v} \nabla \phi, \\
a_{1}=\left|f_{1}\right|_{2}, \quad b_{1}=\left|g_{1}\right|_{2}, \quad a_{2}=\left|f_{2}\right|_{2}, \quad b_{2}=\left|g_{2}\right|_{2} .
\end{gathered}
$$

By the Cauchy-Schwarz inequality we have

$$
\begin{aligned}
(2-\lambda)^{1 / 2}|E-F| & =(2-\lambda)^{1 / 2}\left|G_{1}-G_{2}\right| \\
& =\left|\int\left(f_{1} g_{1}-f_{2} g_{2}\right) d x\right| \\
& \leq a_{1} b_{1}+a_{2} b_{2} .
\end{aligned}
$$

But

$$
\begin{aligned}
0 \leq\left(a_{1} b_{2}-a_{2} b_{1}\right)^{2} & =\left(a_{1}^{2}+a_{2}^{2}\right)\left(b_{1}^{2}+b_{2}^{2}\right)-\left(a_{1} b_{1}+a_{2} b_{2}\right)^{2} \\
a_{1} b_{1}+a_{2} b_{2} & \leq \sqrt{2} \sqrt{\left(a_{1}^{2}+a_{2}^{2}\right) / 2} \sqrt{b_{1}^{2}+b_{2}^{2}} \\
& \leq \frac{1}{\sqrt{2}}\left(\frac{1}{2}\left(a_{1}^{2}+a_{2}^{2}\right)+\left(b_{1}^{2}+b_{2}^{2}\right)\right) \\
& =\frac{1}{\sqrt{2}}((2-\lambda) J+B)
\end{aligned}
$$

and thus

$$
(2-\lambda)^{1 / 2}|E-F| \leq \frac{1}{\sqrt{2}}((2-\lambda) J+B)
$$

Using (2.22) we get

$$
\begin{aligned}
-\left(\frac{d W}{d \tau}+\lambda W\right) \geq \sqrt{2(2-\lambda)}|E-F|+2 E-\mu F \\
=F \cdot(\sqrt{2(2-\lambda)}|X-1|+2 X-\mu)
\end{aligned}
$$


with $X=E / F \geq 0$. For either $d \geq 4$ and $\lambda \leq 2$, or $d=3$ and $\lambda \leq 1$, we have $\mu \leq 2$. The right hand side of (2.23) (positive for $X \geq \mu / 2$ ) equals (for $X \leq$ $\mu / 2 \leq 1)$

$$
\sqrt{2(2-\lambda)}(1-X)+2 X-\mu=(2-\sqrt{2(2-\lambda)}) X+\sqrt{2(2-\lambda)}-\mu,
$$

so that

$$
\sqrt{2(2-\lambda)} \geq \mu
$$

guarantees $\frac{d W}{d \tau}+\lambda W \leq 0$, which implies (2.21). The condition (2.24) is equivalent to $\lambda \leq \lambda(d)$. In particular, $\lambda(d)$ is an increasing function of $d$, $\lambda(3)=\sqrt{7}-2<1, \lambda(4)=4 \sqrt{3}-6$ and $\lim _{d \rightarrow+\infty} \lambda(d)=\frac{3}{2}$.

Remark 2.4 In the case of one species of particles, i.e. $v \equiv 0$ as was in [3] and [4], the result of Proposition 2.2 still holds.

Finally, we remark that there is, in general, no hope to have $\lambda>2$ in nontrivial cases. This can be inferred from the formula (2.22), where for each $\chi>1, J-\chi\left(\int u \log \left(\frac{u}{u_{\infty}}\right) d x+\int v \log \left(\frac{v}{v_{\infty}}\right) d x\right)$ could be negative and dominate the other terms (for instance, in the limit $M_{u}, M_{v} \rightarrow 0^{+}$).

\section{Proof of Theorem 1.2}

First, we recall that steady states $\langle U, V, \Phi\rangle$ of (1.1)-(1.3) satisfy the relations

$$
\nabla \cdot\left(e^{-\Phi} \nabla\left(e^{\Phi} U\right)\right)=0, \quad \nabla \cdot\left(e^{\Phi} \nabla\left(e^{-\Phi} V\right)\right)=0,
$$

hence

$$
U=M_{u} \frac{e^{-\Phi}}{\int e^{-\Phi} d x}, \quad V=M_{v} \frac{e^{\Phi}}{\int e^{\Phi} d x} .
$$

Together with (1.3) this leads to the Poisson-Boltzmann equation

$$
\Delta \Phi=M_{v} \frac{e^{\Phi}}{\int e^{\Phi} d x}-M_{u} \frac{e^{-\Phi}}{\int e^{-\Phi} d x} .
$$

This equation, supplemented with the Dirichlet boundary condition (1.6) or the free condition (1.7), for every $M_{u}, M_{v} \geq 0$, has a unique (weak) solution 
$\Phi$, see [9] or Proposition 2 in [5] (and this solution is classical whenever $\partial \Omega$ is of class $C^{1+\epsilon}$ for some $\left.\epsilon>0\right)$.

The evolution of the Lyapunov functional defined by (1.16) in the case of the Dirichlet boundary condition (1.6) or in the case (1.7) is given by

$$
\frac{d W}{d t}=-\int u|\nabla(\log u+\phi)|^{2} d x-\int v|\nabla(\log v-\phi)|^{2} d x,
$$

cf. (35) in [5], where the above relation is obtained for weak solutions to the Debye-Hückel system.

Concerning the global in time existence of solutions to the Debye-Hückel system with nonlinear boundary conditions (1.4)-(1.5), we note that this was proved for $d=2$ only in Theorem 3 of [5]. Thus, in higher dimensions $d \geq 3$, we assume that $\langle u(t), v(t)\rangle$ exists for all $t \geq 0$. If equations (1.1)-(1.3) are supplemented with linear type boundary conditions (as it is the case in semiconductor modelling), the assumption $u_{0}, v_{0} \in L^{r}(\Omega)$ with an exponent $r>d / 2$ (cf. Theorem 2 (ii) in [5] and [1] for the case of the whole space $\mathbb{R}^{d}$ ) guarantees the existence of $\langle u(t), v(t)\rangle$ for all $t \geq 0$.

First, we represent the entropy production terms in (3.3) as

$$
\int u\left|\nabla\left(\log \left(u e^{\phi}\right)\right)\right|^{2} d x=\int u e^{\phi}\left|\nabla\left(\log \left(u e^{\phi}\right)\right)\right|^{2} \frac{e^{-\phi}}{\int e^{-\phi} d x} d x \cdot \int e^{-\phi} d x,
$$

with an obvious modification for the second term. Then we recall Remark 3.7 of [2], where counterparts of the logarithmic Sobolev inequality (2.15) (or Poincaré-type inequalities) are discussed in the case of a bounded uniformly convex domain. We apply this remark to the domain $\Omega$ and the probability measure

$$
\rho_{0}(x)=\frac{e^{-\phi}}{\int e^{-\phi} d x}
$$

in the first entropy production term in (3.3) written as in (3.4). This implies the existence of a constant $C(\Omega)>0$ such that

$$
\int \Psi\left(\frac{f}{\int f d \rho_{0}}\right) d \rho_{0} \leq C(\Omega) \int \Psi^{\prime \prime}\left(\frac{f}{\int f d \rho_{0}}\right) \frac{|\nabla f|^{2}}{\left(\int f d \rho_{0}\right)^{2}} d x
$$

where $\Psi(s)=1-s+s \log s$ and $f=u e^{\phi}$. Here we have

$$
\int u\left|\nabla\left(\log \left(u e^{\phi}\right)\right)\right|^{2} d x=M_{u} \int \Psi^{\prime \prime}\left(\frac{f}{\int f d \rho_{0}}\right) \frac{|\nabla f|^{2}}{\left(\int f d \rho_{0}\right)^{2}} d x
$$


since $\int f d \rho_{0}=\int u e^{\phi} d \rho_{0}=\frac{M_{u}}{\int e^{-\phi} d x}$. Thus we arrive at

$$
\int u\left|\nabla\left(\log \left(u e^{\phi}\right)\right)\right|^{2} d x \geq \frac{M_{u}}{C(\Omega)} \int\left(\frac{f}{\int f d \rho_{0}} \log \left(\frac{f}{\int f d \rho_{0}}\right)+1-\frac{f}{\int f d \rho_{0}}\right) d \rho_{0},
$$

or

$$
\int u\left|\nabla\left(\log \left(u e^{\phi}\right)\right)\right|^{2} d x \geq \frac{1}{C(\Omega)} \int u \log \left(\frac{u e^{\phi}}{\frac{M_{u}}{\int e^{-\phi} d x}}\right) d x
$$

Similarly, we have

$$
\int v\left|\nabla\left(\log \left(v e^{-\phi}\right)\right)\right|^{2} d x \geq \frac{1}{C(\Omega)} \int v \log \left(\frac{v e^{-\phi}}{\int M_{v}}\right) d x .
$$

Now we compute the expression

$$
\delta=\int u \log \left(\frac{u e^{\phi}}{\frac{M_{u}}{\int e^{-\phi} d x}}\right) d x+\int v \log \left(\frac{v e^{-\phi}}{\frac{M_{v}}{\int e^{\phi} d x}}\right) d x
$$

If $\langle U, V, \Phi\rangle$ is the solution of the Poisson-Boltzmann equation (3.2) with the homogeneous Dirichlet boundary conditions, then it can be checked that

$$
\delta=W+J[\phi]-J[\Phi]
$$

where

$$
\begin{aligned}
W & =\int u \log u d x+\int v \log v d x+\frac{1}{2} \int|\nabla \phi|^{2} d x \\
& -\int U \log U d x-\int V \log V d x-\frac{1}{2} \int|\nabla \Phi|^{2} d x
\end{aligned}
$$

is as in (1.16), and

$$
J[\phi]=\frac{1}{2} \int|\nabla \phi|^{2} d x+M_{u} \log \left(\int e^{-\phi} d x\right)+M_{v} \log \left(\int e^{\phi} d x\right)
$$

is a strictly convex functional reaching its minimum at $\Phi$. 
Now it is clear from (3.3), (3.5)-(3.6) and (3.7) that for some $\lambda=\lambda(\Omega)>0$ $\frac{d W}{d t}+\lambda W \leq 0$, i.e. $W(t)$ decays exponentially in $t$

$$
W(t) \leq W(0) e^{-\lambda t} .
$$

By the Csiszár-Kullback inequality (as was in Section 2), $W(t)$ controls the $L^{1}$-convergence to the unique steady state, so the conclusion (1.19) of Theorem 1.2 follows from (3.8). This improves (34) in Theorem 6 of [5] in two ways. First, there is an exponential decay rate. Second, (34) is proved under the assumption $W(0)<\infty$, which is much weaker than the assumption on the $L^{2}$-boundedness in time of the solution $\langle u, v\rangle$ in Theorem 6 of [5]. Evidently, this result is also valid for one species case $\left(M_{u}\right.$ or $M_{v}$ equal to 0$)$, so Theorem 2 in [3] is also improved.

Acknowledgements. This research was partially supported by the grants POLONIUM 98111 and KBN 324/P03/97/12. The second author thanks the program on Charged Particle Kinetics at the Erwin Schroedinger Institute and the TMR Kinetic Equations for partial support too.

\section{References}

[1] A. Arnold, P. Markowich, G. Toscani, On large time asymptotics for drift-diffusion Poisson systems, preprint ESI no. 655 (1999), 1-11.

[2] A. Arnold, P. Markowich, G. Toscani, A. Unterreiter, On logarithmic Sobolev inequalities, Csiszár-Kullback inequalities, and the rate of convergence to equilibrium for Fokker-Planck type equations, preprint TMR "Asymptotic Methods in Kinetic Theory" no. 12 (1998), 1-77.

[3] P. Biler, Existence and asymptotics of solutions for a parabolic-elliptic system with nonlinear no-flux boundary conditions, Nonlinear Analysis T.M.A. 19 (1992), 1121-1136.

[4] P. Biler, The Cauchy problem and self-similar solutions for a nonlinear parabolic equation, Studia Math. 114 (1995), 181-205.

[5] P. Biler, W. Hebisch, T. Nadzieja, The Debye system: existence and large time behavior of solutions, Nonlinear Analysis T.M.A. 23 (1994), 1189-1209. 
[6] I. Csiszár, Information-type measures of difference of probability distributions and indirect observations, Studia Sci. Math. Hungar. 2 (1967), 299-318.

[7] J. Dolbeault, M. del Pino, Generalized Sobolev inequalities and asymptotic behaviour in fast diffusion and porous medium problems, preprint Ceremade no. 9905 / ESI no. 704 (1999), 1-42.

[8] J. Dolbeault, G. Rein, Time-dependent rescalings and Lyapunov functionals for the Vlasov-Poisson and Euler-Poisson systems, and for related models of kinetic equations, fluid dynamics and quantum physics, preprint Ceremade no. 9945 / TMR "Asymptotic Methods in Kinetic Theory" no. 102 (1999), 1-34.

[9] D. Gogny, P.-L. Lions, Sur les états d'équilibre pour les densités électroniques dans les plasmas, RAIRO Modél. Math. Anal. Numér. 23 (1989), 137-153.

[10] S. Kullback, A lower bound for discrimination information in terms of variation, IEEE Trans. Information Theory 13 (1967), 126-127.

[11] G. Toscani, Sur l'inégalité logarithmique de Sobolev, C. R. Acad. Sci. Paris Sér. I Math. 324 (1997), 689-694. 